\title{
Multilayered dipolar particles in an external magnetic field
}

\author{
Ludovic Spiteri, Hervé Mohrbach, and René Messina* \\ Université de Lorraine, Laboratoire de Physique et Chimie Théoriques, LPCT - UMR CNRS 7019, 1 Boulevard Arago, 57070 Metz, France
}

(Received 1 February 2019; revised manuscript received 16 April 2019; published 25 September 2019)

\begin{abstract}
The effect of an external perpendicular magnetic field on the magnetization and crystallization of magnetic particles trapped in multilayered arrays is investigated theoretically. General ground-state symmetry considerations corroborated by numerical results allow us to develop an exact analytical theory. When dealing with monolayers, the continuous degeneracy of the in-plane dipole orientation is broken and accompanied by a rhombic crystal structure at finite applied magnetic field. On the other hand, bilayer systems are more robust against applied magnetic fields and preserve the in-plane hexagonal lattice symmetry. The onset of disaggregation is predicted as well in both scenarios. Our findings can be experimentally verified in colloidal suspensions (typically ferrofluids or magnetorheological fluids) and provide enlightening hints to further understanding the phase behavior of magnetic multilayers in more conventional molecular and atomic materials such as thin metal films obtained by epitaxy in the presence of an external magnetic field.
\end{abstract}

DOI: 10.1103/PhysRevResearch.1.023028

\section{INTRODUCTION}

Two-dimensional dipolar systems (monolayers or fewlayer systems) are a fertile area of research [1-3]. Some experimental examples are (thin) magnetic films [1,4,5], magnetic bubbles in garnet films [6,7], and magnetic particles on a substrate [8-12] or floating on the surface or the meniscus of a liquid [13-15]. Such systems can benefit the design of novel materials (e.g. photonic band-gap [16] and data storage devices [17]) and can also act as models to better understand certain biological mechanisms and processes (e.g., microtubule [18] and lipid layer formations [19]). Dipolar monolayers are known to exhibit a rich and intricate phase behavior around the chaining regime at finite temperature as observed in computer simulations [2,20-24] and in experiments on ferrofluids $[25,26]$. Formation of multilayers in ferrofluids with smectic-like ordering was also reported experimentally [27] and by computer simulations [28]. In a pioneering theoretical work, the ground state of a planar rhombic lattice with an arbitrary rhombicity angle has been addressed [29,30]. In particular, the influence of an (imposed) lattice symmetry on the resulting dipole moment distribution (in the ground state) was advocated. A related recent study involving an interaction cutoff confirmed the latter findings [31]. Conversely, under a tilting magnetic field, many structural transitions are induced in a two-dimensional superparamagnetic suspension depending on the field strength, the tilt angle, and the density [32]. In our study where we have to deal with permanent dipole moments (i.e., ferromagnetic particles), their strength remains

\footnotetext{
*rene.messina@univ-lorraine.fr

Published by the American Physical Society under the terms of the Creative Commons Attribution 4.0 International license. Further distribution of this work must maintain attribution to the author(s) and the published article's title, journal citation, and DOI.
}

unchanged under an applied magnetic field. Moreover, in the absence of an external magnetic field, the ground state of a monolayer is the hexagonal lattice with a continuous degeneracy of the orientation of the (purely) in-plane dipole moment $[30,33]$. We offer a transparent rederivation of this intriguing result; see Appendix A.

In this paper, we address the effect of an external perpendicular magnetic field on the magnetization and crystallization of attractive magnetic particles trapped in multilayered arrays. It is worthwhile recalling that a tangential magnetic field has no incidence on the hexagonal ground-state layered lattices [34]. In the scope of this article and for the sake of clarity, we shall only focus on the relevant perpendicular magnetic field situation. We argue that dipolar hard sphere systems (i) provide unbiased insight into the intricate interplay between steric effects and long-range anisotropic dipolar interactions and (ii) are also of interest in their own right. Preliminary general symmetry considerations corroborated by numerical optimization will allow us to build a robust exact analytical approach whose details are provided in the Appendix. In this paper, we present a theoretical approach treating multilayered dipolar hard spheres in an external magnetic field [35].

The article is organized as follows. Section II is devoted to the description of the model. Results for the monolayer case are presented in Sec. III. Results for bilayers and implications for multilayers are discussed in Sec. IV. Concluding remarks including a discussion about experiments are provided in Sec. V.

\section{MODEL}

Crystal layered structures made up of dipolar particles are illustrated in Fig. 1. The constitutive dipolar particles are hard spheres of diameter $d$ that sets the unit of length. They each possess a dipole moment of identical magnitude $\|\vec{m}\|=m$ set to unity. The presence of the external normal magnetic 

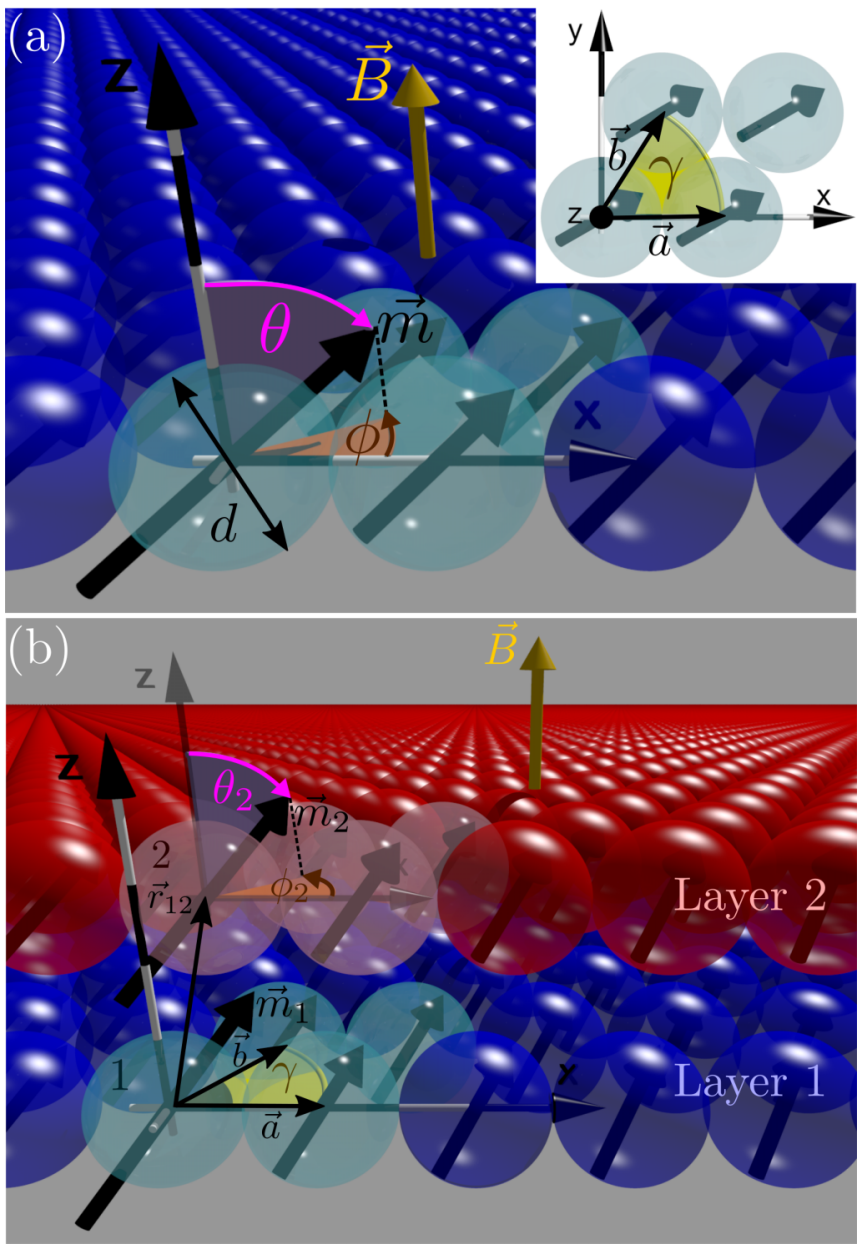

FIG. 1. (a) Sketch of the geometric setup for an infinite monolayer in an external magnetic field. A monoatomic unit cell (light spheres) with its replica (dark spheres) are shown. To better illustrate the dipole orientation angles $(\theta, \phi)$, one dipole moment $\vec{m}$ was magnified. A top view of the unit cell is provided as an inset. For the sake of clarity, we have not shown the more generic diatomic monolayer. (b) Sketch of the geometric setup for an infinite bilayer in an external magnetic field. A diatomic unit cell (light spheres) with its replica (dark spheres) are shown. Layers 1 and 2 are characterized by the atomic dipole moments $\vec{m}_{1}$ and $\vec{m}_{2}$, respectively. For the sake of clarity, only the orientation angles $\left(\theta_{2}, \phi_{2}\right)$ of $\vec{m}_{2}$ are shown.

field, $\vec{B}=B \vec{e}_{z}$ (with $\vec{e}_{z}$ standing for the unit vector along the $z$ axis), involves the Zeeman term $-\vec{m} \cdot \vec{B}\left(=-m_{z} B\right)$. Hence, in general, for a prism unit cell containing $N$ dipolar particles located at $\vec{r}_{1}, \ldots, \vec{r}_{N}$, the dipolar energy per unit cell, $U_{\text {cell }}$, follows

$$
\begin{aligned}
U_{\text {cell }}= & \frac{1}{2} \sum_{i, j}^{N} \sum_{\vec{n}}^{\prime} \frac{C}{\left|\vec{r}_{i j}+\stackrel{\leftrightarrow}{t} \cdot \vec{n}\right|^{3}}\left[\vec{m}_{i} \cdot \vec{m}_{j}\right. \\
& \left.-3 \frac{\vec{m}_{i} \cdot\left(\vec{r}_{i j}+\stackrel{\leftrightarrow}{t} \cdot \vec{n}\right) \vec{m}_{j} \cdot\left(\vec{r}_{i j}+\stackrel{\leftrightarrow}{t} \cdot \vec{n}\right)}{\left|\vec{r}_{i j}+\overleftrightarrow{t} \cdot \vec{n}\right|^{2}}\right]-\sum_{i=1}^{N} m_{i, z} B,
\end{aligned}
$$

where $\stackrel{\leftrightarrow}{t}$ is a $2 \times 2$ matrix whose two columns are given by the spanning vectors $\vec{a}=(a, 0)$ and $\vec{b}=\left(b_{x}, b_{y}\right)$ in the $(x, y)$ plane. $\vec{n}$ in Eq. (1) designates a vector with integer components $\left(n_{x}, n_{y}\right)$, each ranging from $-\infty$ to $+\infty$. The prime in Eq. (1) indicates that when $\vec{n}=0, i$ must be different from $j$. The energy scale is set by $U_{\uparrow \uparrow} \equiv C m^{2} / d^{3}$ with $C=\frac{\mu_{0}}{4 \pi}$. Thereby, the reduced dipolar energy per particle, $u$, is merely given by $u=\frac{1}{N} \frac{U_{\text {cell }}}{U_{\uparrow \uparrow}}$. In what follows, the magnetic field $B$ will always be in units of $U_{\uparrow \uparrow} / m$.

On the numerical computational side, in order to overcome the slow (conditional) convergence [36] in Eq. (1), an efficient Ewald sum technique adapted to the parallelogram base with periodicity in two directions was used [37]. Ground states are obtained by minimization procedures starting with a generic parallelogram cell made up of two particles. Thus, for the onelayer case, the minimization of $u$ at a prescribed magnetic field involves nine parameters corresponding to the two modules of spanning vectors $a$ and $b$, the angle $\gamma=\widehat{(\vec{a}, \vec{b}})$, the two Cartesian coordinates of the second constitutive particle, and the four related dipole orientation angles, see also Fig. 1(a). In the two-layer situation, an additional variable sets in which is the transverse shift between the two layers, see also Fig. 1(b). The efficient SLSQP (sequential least square programming) method is employed, which natively takes constraints into account and requires derivatives of the objective function [38]. Typically $2 \times 10^{3}$ starting random configurations were needed to reach the global minimum.

\section{MONOLAYER IN A NORMAL MAGNETIC FIELD}

\section{A. Numerical results}

Results about the monolayer are now presented. The numerical calculations unambiguously indicate that the unit cell of the crystal ground state is always rhombic $(a=b=1)$ and monoatomic $(N=1)$. Thereby, the angle $\gamma$ will be referred to as the rhombicity angle. A major finding is the slight but relevant symmetry breaking of the hexagonal lattice upon applying an external magnetic field, i.e., $\gamma>60^{\circ}$ when $B>$ 0 . This intriguing result can be inspected in Fig. 2(a), where $\gamma(B)$ is plotted. Clearly, the deviation from the hexagonal lattice increases monotonically with the magnetic field $B$, see Fig. 2(a). An important physical consequence is that the continuous degeneracy of the in-plane dipole $\vec{m}_{\rho}=\vec{m}-m_{z} \vec{e}_{z}$, existing in a perfect hexagonal lattice $\left(\gamma=60^{\circ}\right)$ [30], is now suppressed in the presence of an external magnetic field that induces rhombicity $\left(\gamma>60^{\circ}\right)$. Concomitantly, the azimuthal angle $\phi$ of the dipole corresponds to $\gamma / 2$ in agreement with past studies without an external magnetic field [30,31]. The related profile of the induced magnetization $m_{z}(B)$ (which also corresponds to $\cos \theta$ ) is displayed in Fig. 2(b). It exhibits a quasilinear law and reaches a value of the order of $20 \%$ around the onset of disaggregation (here at $B=B_{\mathrm{tr}}=3.04$ ), see Fig. 2(b). The latter merely sets in when the decohesion energy overcomes the Zeeman term, see also Eq. (2), which is discussed in the next paragraph. The related profile of the ground-state energy as a function of the applied magnetic field $B, u(B)$, can be found in Fig. 2(c). As expected, the cohesive energy, $u_{\text {coh }}=u+m_{z} B$, increases with $B$ (i.e., getting less 
(a)

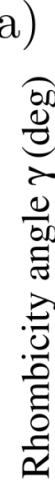

(b)

(c)

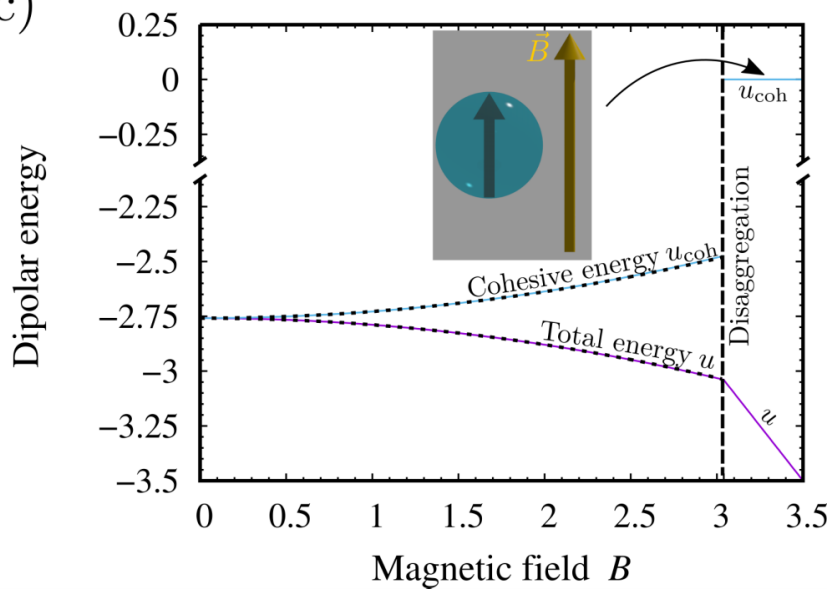

FIG. 2. (a) Rhombicity angle $\gamma$, (b) $z$ component of the dipole moment $m_{z}$, and (c) total and cohesive energy profiles as a function of the external magnetic field intensity $B$ for the monolayer case. Top and side views of the rhombic unit cell are displayed as insets. The solid lines in (a)-(c) correspond to the exact theory in quantitative agreement with the numerical data. The dashed lines in (b) and (c) correspond to the HLA model, see Eq. (5) and text around it. The vertical dashed line in (a)-(c) indicates the disaggregation onset occurring at $B=B_{\mathrm{tr}}=3.04$. For $B>B_{\mathrm{tr}}$, results for a solitary magnet are shown for reference.

favorable) whereas the total energy $u$ decreases. In the disaggregation regime $B>B_{\mathrm{tr}}$, results for infinite dilution (i.e., solitary magnet) are shown for reference with $u_{\text {coh }}=0$ and $u=-B$, see Fig. 2(c).

\section{B. Analytical approach \\ 1. Exact theory}

Deeper understanding can be gained by solving exactly and analytically the depicted problem of magnetic layers exposed to a magnetic field. It turns out that this formidable challenge is feasible noticing the still strong symmetry of the problem and upon dexterously rewriting the Hamiltonian given by Eq. (1) in a tractable way. In a general fashion, it is insightful to write the dipolar energy $u$ for a (monolayer) rhombic lattice in the following (exact) form:

$$
u=E_{0}(\gamma)+E_{1} m_{z}+E_{2}(\gamma) m_{z}^{2},
$$

with $E_{0}(\gamma)$ representing the cohesive energy of the (magnetically isolated) rhombic lattice with $m_{z}=0, E_{1}=-B$ stemming from the Zeeman term, and $E_{2}(\gamma)>0$ causing the dipolar decohesion. In Appendix A, we provide the full exact analytical and tractable expression of $u\left(m_{z}, \gamma ; B\right)$ obtained at the prescribed magnetic field $B$, see Eqs. (A1)-(A3). Having this at hand, one can extract the magnetization in the ground state stemming from the condition $\left.\frac{\partial u}{\partial m_{z}}\right|_{B}=0$ that trivially yields

$$
m_{z}=\frac{B}{2 E_{2}(\gamma)},
$$

with $\gamma=\gamma(B)$ in Eq. (3). Hence, the susceptibility $\chi$ of the system is merely identical to $\left(2 E_{2}\right)^{-1}$. Similarly, the minimum energy condition requires also simultaneously $\left.\frac{\partial u}{\partial \gamma}\right|_{B}=0$, leading to a highly intricate dependence of $\gamma$ with $B$, see Appendix A for calculation details. The ground state profile of $\gamma(B)$ can be visually inspected in Fig. 2(a). Consequently, the ground state energy of the rhombic phase reads

$$
u(B)=E_{0}(B)-\frac{\chi(B)}{2} B^{2},
$$

whose profile is displayed in Fig. 2(c).

\section{Hexagonal lattice approximation}

Another analytical route which is much more simple, but not exact, is to neglect the rhombicity angle variation, i.e., always assume a hexagonal lattice $\left[\gamma(B)=60^{\circ}\right]$, see Appendix A 2 for details. The latter approach will be referred to as the hexagonal lattice approximation (HLA). In this scenario, the ground-state energy reads

$$
u^{\mathrm{HLA}}=-2.759-3.021 \times 10^{-2} B^{2},
$$

where $E_{0}=-2.759$ corresponds to the well-known cohesive energy of a hexagonal lattice [31,39], see also Eq. (B13), and $\chi=6.042 \times 10^{-2}$ is the related susceptibility, see Appendix A for the full derivation. The profile of $u^{\mathrm{HLA}}(B)$ specified by Eq. (5) is displayed in Fig. 2(c), too, rationalizing also the (quasi) linear behavior found in $m_{z}(B)$, see Fig. 2(b). The various values of $\chi$ and $B_{\mathrm{tr}}$ are gathered in Table I.

\section{BILAYER IN A NORMAL MAGNETIC FIELD}

\section{A. Numerical results}

We now address the bilayer system whose setup is sketched in Fig. 1(b). Interestingly, our numerical optimization predicts that the two layers adopt a hexagonal lattice with identical 
TABLE I. Numerical values of $\chi, B_{\text {tr }}$ and $E_{0}$ in different scenarios.

\begin{tabular}{lccc}
\hline \hline & \multicolumn{2}{c}{ Monolayer } & Bilayer \\
\cline { 2 - 3 } & Exact theory & HLA model & Exact theory \\
\hline$\chi$ & $B$ dependent & $6.042 \times 10^{-2}$ & $5.868 \times 10^{-2}$ \\
$B_{\mathrm{tr}}$ & 3.0391 & 3.0372 & 3.2062 \\
$E_{0}$ & $B$ dependent & -2.759 & -2.905 \\
\hline \hline
\end{tabular}

moments $\left(\vec{m}_{1}=\vec{m}_{2}=\vec{m}\right)$ in the ground state at finite applied external magnetic field. That is to say that the two-layer lattice is invariant upon exerting a magnetic field and coincides with the body-centered tetragonal lattice. The latter lattice structure constitutes a signature of dipolar systems and does not emerge in double-layered charged particles $[40,41]$. The azimuthal angle $\phi$ of the dipole corresponds now to $\gamma$ (i.e., $\phi=\gamma$ ), see also inset of Fig. 3(a). Thereby the bilayer magnetization matches that of the body-centered tetragonal ground state. In contrast with the monolayer case, here there is no longer a continuous degeneracy of the dipole moment orientation.

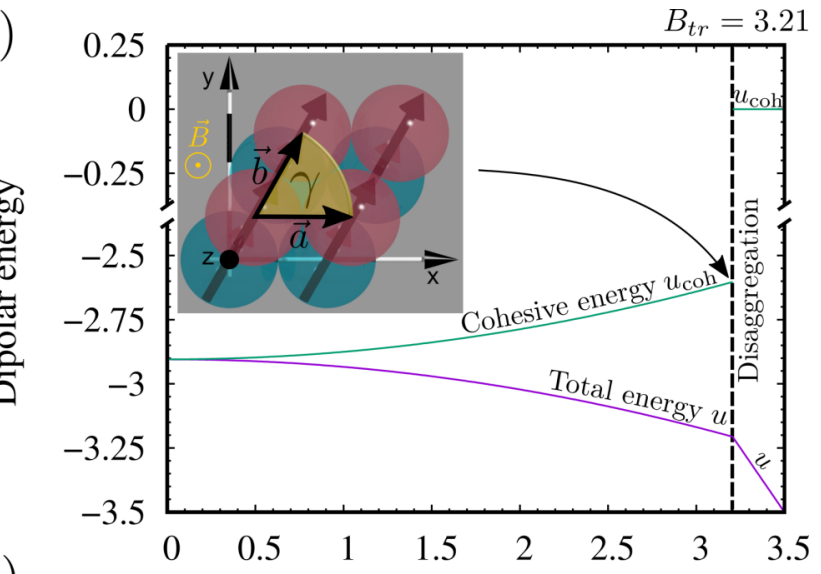

(b)

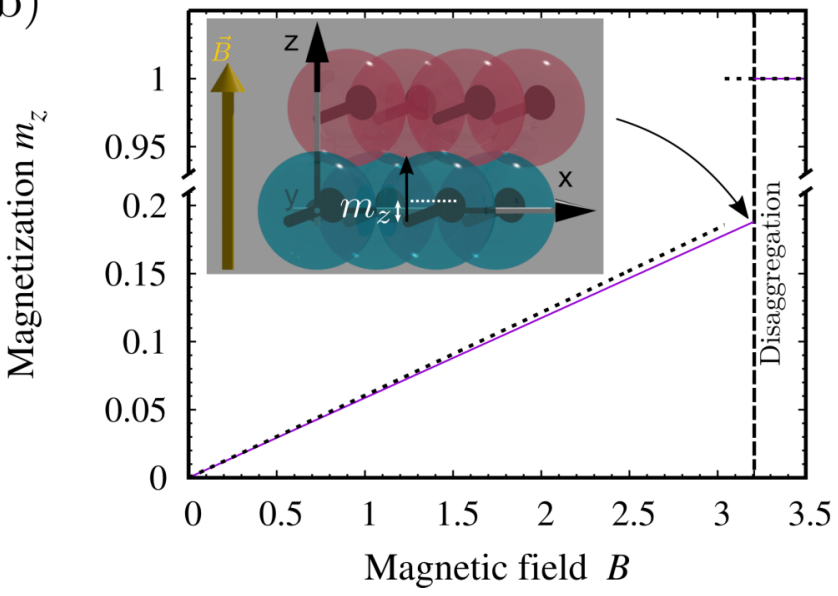

FIG. 3. (a) Total and cohesive energy profiles, see related Eq. (6), and (b) $z$ component of the dipole moment $m_{z}$ as a function of the external magnetic field intensity $B$ for the bilayer, see Eq. (7). The magnetization $m_{z}(B)$ for the monolayer is shown as a dashed line for comparison. Top and side views of the two-layer lattice are displayed as insets in (a) and (b), respectively. For $B>B_{\mathrm{tr}}$, results for a solitary magnet are shown for reference.

\section{B. Exact analytical results}

This strong symmetry preservation allows an exact analytical description too, which is detailed in Appendix B. As a matter of fact, relation (2) gets simplified where the $\gamma$ dependence vanishes. It can be shown, see also Appendix B, that the (exact) dipolar energy $u$ in the ground state verifies

$$
u_{\mathrm{bi}}=-2.905-2.934 \times 10^{-2} B^{2},
$$

whose profile can be found in Fig. 3(a). Notice the high resemblance with the (approximate) monolayer energy $u^{\mathrm{HLA}}$, see Eq. (5). The enhanced cohesion upon stacking (here $E_{0}=$ -2.905 to be compared with $E_{0}=-2.759$ for a monolayer in the absence of an applied magnetic field) leads to two notable features:

(i) A lower susceptibility emerges, see Appendix B for derivation, where

$$
m_{z}^{\mathrm{bi}}=5.868 \times 10^{-2} B,
$$

whose profile appears in Fig. 3(b); see also Table I.

(ii) The disaggregation onset is shifted to a higher magnetic field, see Fig. 3(a) and also Table I.

It is insightful to recall that in the bulk limit the cohesion energy is $E_{0}=-3.05[42,43]$, which is already close to that of the bilayer $E_{0}=-2.905$ (i.e., about $5 \%$ ).

\section{CONCLUDING REMARKS}

In summary, we have investigated theoretically the ground state of magnetic particles trapped in multilayered arrays exposed to a perpendicular external magnetic field. The monolayer ground state is found to be always rhombic. Without an external magnetic field, the well-known hexagonal lattice with a continuously degenerate ferromagnetic state is recovered as the ground state [30]. At a finite external field, the hexagonal lattice symmetry and the related continuous degeneracy are broken. In strong contrast and interestingly, the ground-state structure of the bilayer system is invariant under an applied perpendicular magnetic field. At finite external magnetic field, the lattice of each layer is hexagonal and the composite superlattice of the bilayer coincides with the body-centered tetragonal lattice [42-45]. Concomitantly, the magnetic susceptibility is reduced due to the enhanced cohesion upon stacking a second layer.

It is therefore expected that multilayers (beyond bilayers) consist of stacked hexagonal layers coinciding with the bodycentered tetragonal structure. Addressing more quantitatively (true) multilayers is possible, albeit very cumbersome analytically, and is planned in a future study. An interesting question would be to estimate how fast the induced magnetization saturates towards the bulk value.

On the experimental side, our findings are relevant too and can be verified in various settings. For instance, the so-called magnetic click colloidal self-assembly [46] can be further explored (in the dense layer regime) by applying an external perpendicular magnetic field and examining the induced disaggregation. As far as magnetic multilayers are concerned, our findings can be verified and exploited in multilayered photonic crystals made up of centimetric permanent magnets floating at the air-liquid interface $[47,48]$. At the mesoscopic scale, magnetic colloidal multilayers should be in principle 


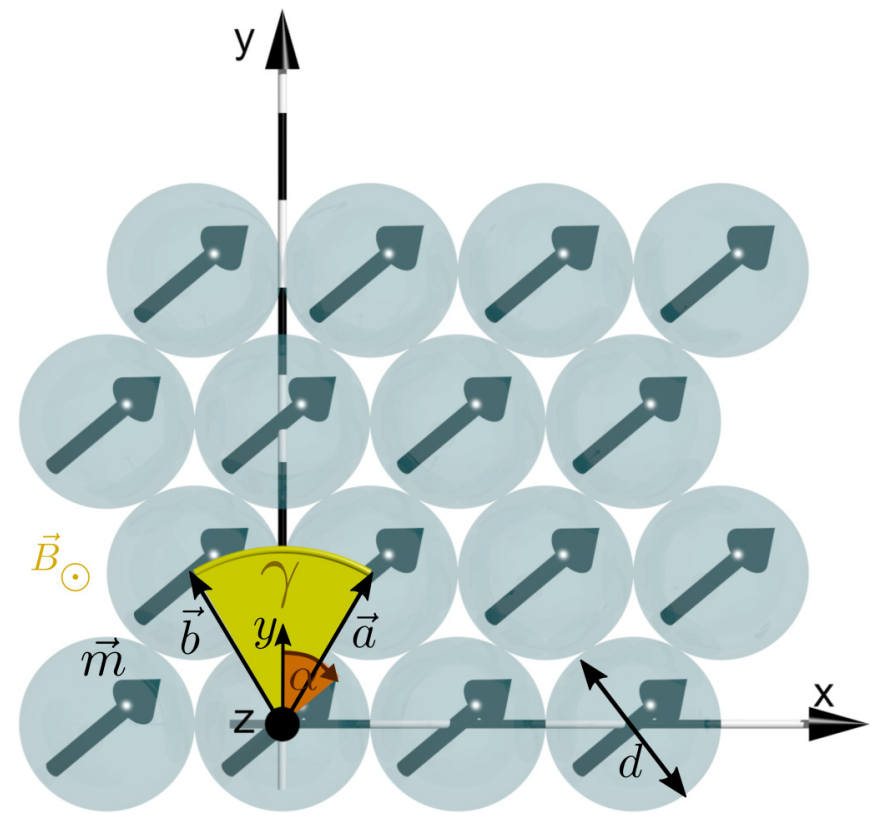

FIG. 4. Sketch of the geometric setup for an infinite monolayer in a perpendicular magnetic field where the bisector of $\widehat{(\vec{a}, \vec{b})}$ coincides with the $y$ axis.

achievable at air-water [49] or solid-water [50] interfaces. As a matter of fact, strong smectic-like ordering has been experimentally observed in ferrofluids at silica interfaces when exposed to a normal magnetic field [27]. In this respect, our study sheds light on the lateral ordering and resulting magnetization in such systems. On a final note, we would like to mention the striking analogy between the magnetic field induced rhombicity and subsequent disaggregation reported in our work and the so-called Rosensweig instability [51]. In this magnetohydrodynamic instability occurring at a ferrofluid interface exposed to a critical normal magnetic field, there is a transition from a flat interface to stationary waves with hexagonal patterns [51] and then square patterns [52] upon increasing the magnetic field.

\section{APPENDIX A: MONOLAYER CASE}

When we have considered the case of a monolayer in an external perpendicular magnetic field, preliminary numerical minimization has revealed two major symmetries: (i) The monolayer's lattice is always rhombic (i.e., $\|\vec{a}\|=\|\vec{b}\|$ ) and (ii) the phase is ferromagnetic. To obtain an analytical description of the problem, it is useful to choose the $y$ axis (or equivalently the $x$ axis) to coincide with the bisector of $(\vec{a}, \vec{b})$, see Fig. 4. Thereby, the dipole moment $\vec{m}$ forms an angle $\alpha$ with the $y$ axis, so that $\vec{m}=\left(m_{\rho} \sin \alpha, m_{\rho} \cos \alpha, m_{z}\right)$ with $m^{2}=m_{\rho}^{2}+m_{z}^{2}=1$, see Fig. 4. Within this framework and after some lengthy algebra, one arrives at the following exact expression for the energy per particle at prescribed rhombicity $\gamma$ :

$$
\begin{aligned}
u\left(m_{z}, \gamma, \alpha ; B\right)= & S_{1}(\gamma)+\cos (2 \alpha) S_{2}(\gamma)\left(1-m_{z}^{2}\right) \\
& -3 S_{1}(\gamma) m_{z}^{2}-B m_{z},
\end{aligned}
$$

with

$$
\begin{aligned}
S_{1}(\gamma)= & -\frac{1}{16 \cos ^{3}(\gamma / 2)}\left\{\zeta(3)+\frac{4 \pi^{2}}{3[\tan (\gamma / 2)]^{2}}\right. \\
& +\frac{8 \pi}{\tan (\gamma / 2)} \sum_{n=1}^{\infty} \sum_{k=1}^{\infty}\left[\frac{n}{k} K_{1}(2 \pi \tan (\gamma / 2) n k)\right. \\
& \left.\left.+\frac{n \cos (n \pi)}{k-1 / 2} K_{1}(2 \pi \tan (\gamma / 2) n(k-1 / 2))\right]\right\},
\end{aligned}
$$

and

$$
\begin{aligned}
S_{2}(\gamma)= & -\frac{1}{16 \cos ^{3}(\gamma / 2)}\left\{3 \zeta(3)-\frac{4 \pi^{2}}{3[\tan (\gamma / 2)]^{2}}-8 \pi^{2} \sum_{n=1}^{\infty} \sum_{k=1}^{\infty}\right. \\
& \times\left(n^{2}\left[K_{2}(2 \pi \tan (\gamma / 2) n k)+3 K_{0}(2 \pi \tan (\gamma / 2) n k)\right]\right. \\
& +n^{2} \cos (n \pi)\left[K_{2}(2 \pi \tan (\gamma / 2) n(k-1 / 2))\right. \\
& \left.\left.\left.+3 K_{0}(2 \pi \tan (\gamma / 2) n(k-1 / 2))\right]\right)\right\},
\end{aligned}
$$

where $\zeta(3)$ is a zeta function, and $K_{0}(r), K_{1}(r)$, and $K_{2}(r)$ are modified Bessel functions of the second kind. Notice that formula in Eqs. (A2) and (A3) are reminiscent of Lekner-type sums $[53,54]$. However, our lattice sum corresponds to a new suitable formulation adapted to a rhombic lattice.

\section{General expressions for the ground state}

One can show that $S_{2}<0$ with $60^{\circ}<\gamma<120^{\circ}$. Hence, $\alpha=0^{\circ}$ in Eq. (A1) in the ground state. For the hexagonal case with $\gamma=60^{\circ}$ (or equivalently $120^{\circ}$ ), we have $S_{2}=0$ so that $\alpha$ can assume any arbitrary value in the ground state. This provides a more transparent and appealing demonstration (compare with Ref. [30]) of the continuous degeneracy of the orientation of the dipole moment in the hexagonal ground state. In general for the rhombic structure, the total energy per particle of the monolayer, $u_{\text {mono }}$, can be cast into the form

$$
u_{\mathrm{mono}}\left(m_{z}, \gamma ; B\right)=E_{0}^{\mathrm{mono}}(\gamma)+E_{1}^{\mathrm{mono}} m_{z}+E_{2}^{\mathrm{mono}}(\gamma) m_{z}^{2},
$$

with

$$
\begin{aligned}
E_{0}^{\text {mono }}(\gamma)= & S_{1}+S_{2} \\
= & -\frac{1}{4 \cos ^{3}(\gamma / 2)}\left\{\zeta(3)-8 \pi^{2} \sum_{n=1}^{\infty} \sum_{k=1}^{\infty}\right. \\
& \times\left[n^{2} K_{0}(2 \pi \tan (\gamma / 2) n k)\right. \\
& \left.\left.+n^{2} \cos (n \pi) K_{0}(2 \pi \tan (\gamma / 2) n(k-1 / 2))\right]\right\},
\end{aligned}
$$

$$
E_{1}^{\text {mono }}=-B,
$$


$E_{2}^{\text {mono }}(\gamma)=-3 S_{1}-S_{2}$

$$
\begin{aligned}
= & \frac{1}{8 \cos ^{3}(\gamma / 2)}\left\{3 \zeta(3)+\frac{4 \pi^{2}}{3(\tan (\gamma / 2))^{2}}\right. \\
& +8 \pi^{2} \sum_{n=1}^{\infty} \sum_{k=1}^{\infty}\left(n ^ { 2 } \left[K_{2}(2 \pi \tan (\gamma / 2) n k)\right.\right. \\
& \left.-3 K_{0}(2 \pi \tan (\gamma / 2) n k)\right] \\
& +n^{2} \cos (n \pi)\left[K_{2}(2 \pi \tan (\gamma / 2) n(k-1 / 2))\right. \\
& \left.\left.\left.-3 K_{0}(2 \pi \tan (\gamma / 2) n(k-1 / 2))\right]\right)\right\} .
\end{aligned}
$$

By minimizing the energy given by Eq. (A4) with respect to $m_{z}$, i.e., $\left.\frac{\partial u}{\partial m_{z}}\right|_{B}=0$, we obtain the magnetization that reads

$$
m_{z}(\gamma ; B)=\frac{B}{2 E_{2}^{\text {mono }}(\gamma)}
$$

and consequently the total energy given by Eq. (A4) becomes

$$
u_{\text {mono }}(\gamma ; B)=E_{0}^{\text {mono }}(\gamma)-\frac{B^{2}}{4 E_{2}^{\text {mono }}(\gamma)} .
$$

At prescribed magnetic field $B$, further minimization of Eq. (A10) with respect to $\gamma$, i.e., $\left.\frac{\partial u}{\partial \gamma}\right|_{B}=0$, provides a value $\gamma=\gamma^{*}$ [55], leading to the final ground-state energy

$$
u_{\text {mono }}^{\min }(B)=E_{0}^{\text {mono }}\left(\gamma^{*}(B)\right)-\frac{B^{2}}{4 E_{2}^{\text {mono }}\left(\gamma^{*}(B)\right)}
$$

and magnetization

$$
m_{z}(B)=\frac{B}{2 E_{2}^{\text {mono }}\left(\gamma^{*}(B)\right)} .
$$

\section{Hexagonal lattice approximation (HLA)}

If we neglect the rhombicity and always assume a perfect hexagonal lattice for the ground state, Eq. (A4) becomes

$$
u_{\mathrm{mono}}\left(m_{z} ; B\right)=E_{0}^{\mathrm{mono}}\left(60^{\circ}\right)+E_{1}^{\mathrm{mono}} m_{z}+E_{2}^{\mathrm{mono}}\left(60^{\circ}\right) m_{z}^{2} .
$$

With this approximation the corresponding minimal energy reads

$$
u_{\text {mono }}^{\mathrm{HLA}}(B)=E_{0}^{\mathrm{mono}}\left(60^{\circ}\right)-\frac{B^{2}}{4 E_{2}^{\text {mono }}\left(60^{\circ}\right)},
$$

and the related magnetization is

$$
m_{z}(B)=\frac{B}{2 E_{2}^{\text {mono }}\left(60^{\circ}\right)}=\chi B,
$$

$$
\text { with } \chi=\frac{1}{2 E_{2}^{\text {mono }}\left(60^{\circ}\right)}=0.06042 \text {. }
$$

\section{Disaggregation onset}

The critical value of the magnetic field $B_{\text {tr }}$ at which the (dense) crystal layer is energetically beaten by an infinitely dilute gas (or equivalently a crystal layer with diverging lattice constants $a, b \rightarrow \infty)$ is given by the condition

$$
u_{\text {mono }}^{\min }\left(B_{\text {tr }}\right)=u_{\text {gas }}:=-B_{\text {tr }} \text {. }
$$

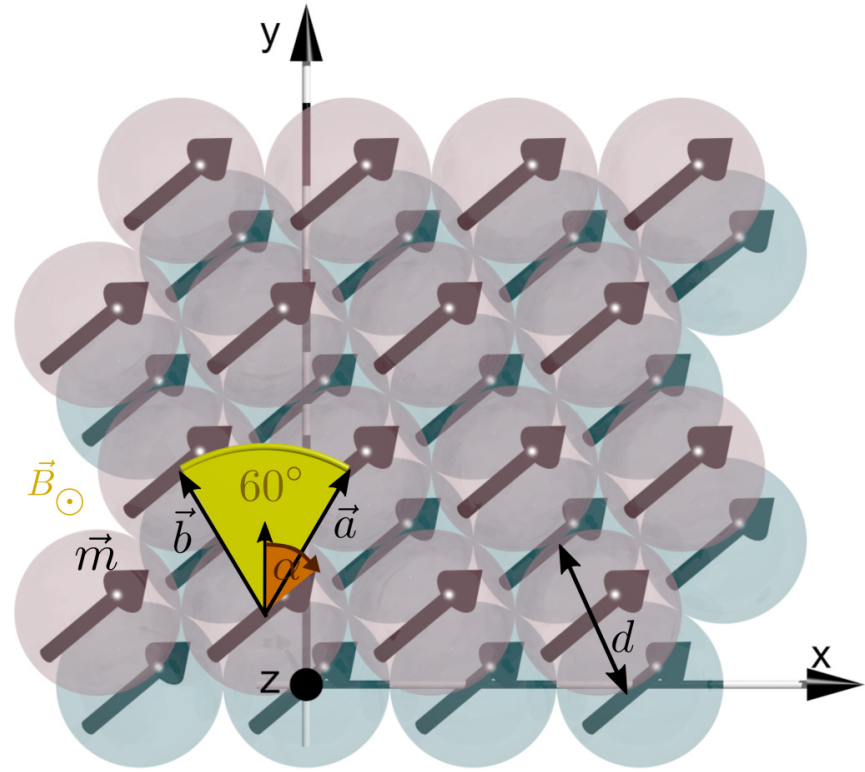

FIG. 5. Sketch of the geometric setup for an infinite bilayer in a perpendicular magnetic field where the bisector of $\widehat{(\vec{a}, \vec{b})}$ parallel to the $y$-axis. The underlying body-centered tetragonal structure is characterized by two staggered hexagonal lattices with a shift of $1 / 2$ in the chaining direction $\left(-30^{\circ}\right.$ with the $y-$ axis coinciding here with $\vec{b}$ ). Note that in this illustration the dipole moments are not relaxed.

By solving Eq. (A16), we find

$$
\begin{gathered}
B_{\mathrm{tr}}=-u_{\mathrm{mono}}^{\min }\left(\gamma_{\mathrm{tr}}^{*}, B_{\mathrm{tr}}\right)=3.039, \\
\gamma_{\mathrm{tr}}^{*} \approx 61.12 .
\end{gathered}
$$

Notice that the energy difference between the deformed lattice and the ideal one is small but not negligible. For instance

$$
\begin{aligned}
& u_{\text {mono }}^{\min }\left(B_{\text {tr }}\right)=-3.0391, \\
& u_{\text {mono }}^{\mathrm{HLA}}\left(B_{\text {tr }}\right)=-3.0376 .
\end{aligned}
$$

\section{APPENDIX B: BILAYER CASE}

In the case of a bilayer in a external perpendicular magnetic field, preliminary numerical minimization has shown that the lattice of each of both constitutive layers is hexagonal (i.e., $\gamma=60^{\circ}$ ) in the ground state, see also Fig. 5. In addition, the bilayer's superlattice coincides with the body-centered tetragonal lattice and the phase is ferromagnetic, see also Fig. 5. Similarly to the monolayer case, we choose the $y$ axis (or equivalently $x$ axis) parallel to the bisector of $(\widehat{\vec{a}, \vec{b}})$, see Fig. 5. Within this framework, the energy per particle, $u_{\mathrm{bi}}$, reads

$$
u_{\mathrm{bi}}\left(m_{z}, \alpha ; B\right)=u_{\text {mono }}\left(m_{z} ; B\right)+u_{\text {inter }}\left(m_{z}, \alpha ; B\right),
$$


where $u_{\text {mono }}\left(m_{z} ; B\right)$ is given by Eq. (A13) and the energy between the two interacting layers, $u_{\text {inter }}$, is

$$
u_{\text {inter }}\left(m_{z} ; B\right)=-\frac{1}{2}\left[I_{1}+I_{2}(\alpha)\right],
$$

with

$$
\begin{aligned}
I_{1}= & \frac{32 \pi}{3 \sqrt{3}} \sum_{k=-\infty}^{\infty} \sum_{n=1}^{\infty} \frac{n \cos \left(\frac{3 n \pi}{2}\right)}{\sqrt{1+\frac{1}{3}\left(2 k-\frac{3}{2}\right)^{2}}} \\
& \times K_{1}\left(n \pi \sqrt{1+\frac{1}{3}\left(2 k-\frac{3}{2}\right)^{2}}\right)+\frac{8}{3} \pi \tanh (\sqrt{3} \pi),
\end{aligned}
$$

and

$$
\begin{aligned}
I_{2}(\alpha)= & -3\left[3\left(1-m_{z}^{2}\right) \cos ^{2}(\alpha) D_{1}+\frac{\left(1-m_{z}^{2}\right) \sin ^{2}(\alpha)}{4} D_{2}\right. \\
& \left.+\frac{3 m_{z}^{2}}{4} D_{3}-\frac{\sqrt{3}\left(1-m_{z}^{2}\right)}{2} \sin (2 \alpha) D_{4}\right]
\end{aligned}
$$

where

$$
\begin{aligned}
& D_{1}=\frac{32 \pi}{27 \sqrt{3}} \sum_{k=-\infty}^{\infty} \sum_{n=1}^{\infty} \\
& \times \cos \left(\frac{n \pi}{2}\right)\left[-n^{2} \pi K_{0}\left(n \pi \sqrt{1+\frac{1}{3}\left(2 k-\frac{1}{2}\right)^{2}}\right)\right. \\
& \left.+\frac{n K_{1}\left(n \pi \sqrt{1+\frac{1}{3}\left(2 k-\frac{1}{2}\right)^{2}}\right)}{\sqrt{1+\frac{1}{3}\left(2 k-\frac{1}{2}\right)^{2}}}\right]+\frac{8 \pi}{27} \tanh (\sqrt{3} \pi), \\
& D_{2}=\frac{128 \pi^{2}}{27 \sqrt{3}} \sum_{k=-\infty}^{\infty} \frac{\left(2 k-\frac{1}{2}\right)^{2}}{1+\frac{1}{3}\left(2 k-\frac{1}{2}\right)^{2}} \\
& \times \sum_{n=1}^{\infty} n^{2} \cos \left(\frac{n \pi}{2}\right) K_{2}\left(n \pi \sqrt{1+\frac{1}{3}\left(2 k-\frac{1}{2}\right)^{2}}\right) \\
& +\frac{16 \pi}{9 \sqrt{3}}[6 \pi+\sqrt{3} \sinh (2 \sqrt{3} \pi)] \operatorname{sech}^{2}(\sqrt{3} \pi), \\
& D_{3}=\frac{128 \pi^{2}}{27 \sqrt{3}} \sum_{k=-\infty}^{\infty} \frac{1}{1+\frac{1}{3}\left(2 k-\frac{1}{2}\right)^{2}} \\
& \times \sum_{n=1}^{\infty} n^{2} \cos \left(\frac{n \pi}{2}\right) K_{2}\left(n \pi \sqrt{1+\frac{1}{3}\left(2 k-\frac{1}{2}\right)^{2}}\right) \\
& +\frac{32 \pi}{27 \sqrt{3}}\left[-3 \pi \operatorname{sech}^{2}(\sqrt{3} \pi)+\sqrt{3} \tanh (\sqrt{3} \pi)\right],
\end{aligned}
$$

TABLE II. Numerical values of some constants appearing in Appendix B.

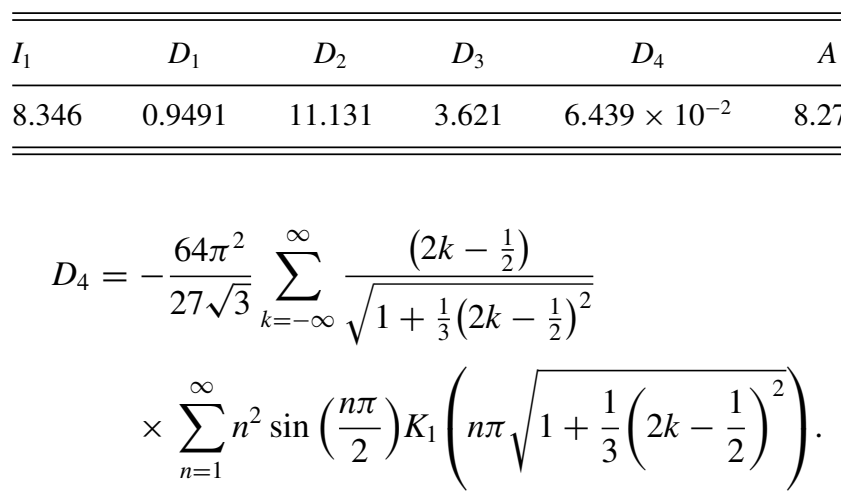

The numerical values of $I_{1}, D_{1}, D_{2}, D_{3}, D_{4}$ defined through Eqs. (B3)-(B8) are gathered in Table II.

\section{General expressions for the ground state}

The minimization of the energy given by Eq. (B1) with respect to the angle $\alpha$, i.e., $\left.\frac{\partial u_{\mathrm{bi}}}{\partial \alpha}\right|_{B}=0$, leads to the value $\alpha=$ $-30^{\circ}$ and consequently the total energy becomes

$$
u_{\mathrm{bi}}\left(m_{z} ; B\right)=E_{0}^{\mathrm{bi}}+E_{1}^{\mathrm{bi}} m_{z}+E_{2}^{\mathrm{bi}} m_{z}^{2},
$$

where

$$
\begin{gathered}
E_{0}^{\mathrm{bi}}=E_{0}^{\mathrm{mono}}\left(60^{\circ}\right)+\frac{1}{2} I_{1}-\frac{3}{4}\left(D_{4}+6 D_{1}\right), \\
E_{1}^{\mathrm{bi}}=-B, \\
E_{2}^{\mathrm{bi}}=\frac{3}{8}\left(9 D_{1}+\frac{1}{4} D_{2}-3 D_{3}+3 D_{4}\right)+A .
\end{gathered}
$$

The term $E_{0}^{\text {mono }}\left(60^{\circ}\right)$ in Eq. (B10), see also Eq. (A5), reads

$$
\begin{aligned}
E_{0}^{\mathrm{mono}}\left(60^{\circ}\right)= & -\frac{1}{3 \sqrt{3}}\left\{2 \zeta(3)-16 \pi^{2} \sum_{n=1}^{\infty} \sum_{k=1}^{\infty} n^{2}\left[K_{0}\left(\frac{2 \pi}{\sqrt{3}} n k\right)\right.\right. \\
& \left.\left.+\cos (n \pi) K_{0}\left(\frac{2 \pi}{\sqrt{3}} n(k-1 / 2)\right)\right]\right\}, \quad \text { (B13) }
\end{aligned}
$$

and the term $A$ in Eq. (B12) is

$$
\begin{aligned}
A= & \frac{1}{3 \sqrt{3}}\left\{3 \zeta(3)+4 \pi^{2}+8 \pi^{2} \sum_{n=1}^{\infty} \sum_{k=1}^{\infty} n^{2}\left[K_{2}\left(\frac{2 \pi}{\sqrt{3}} n k\right)\right.\right. \\
& +\cos (n \pi) K_{2}\left(\frac{2 \pi}{\sqrt{3}} n(k-1 / 2)\right)-3 K_{0}\left(\frac{2 \pi}{\sqrt{3}} n k\right) \\
& \left.\left.-3 \cos (n \pi) K_{0}\left(\frac{2 \pi}{\sqrt{3}} n(k-1 / 2)\right)\right]\right\} .
\end{aligned}
$$

Then, by minimizing the energy established in Eq. (B9) with respect to $m_{z}$, i.e., $\left.\frac{\partial u_{\mathrm{bi}}}{\partial m_{z}}\right|_{B}=0$, we obtain the following magnetization:

$$
m_{z}(B)=\frac{B}{2 E_{2}^{\mathrm{bi}}}=\chi B,
$$

with $\chi=\frac{1}{2 E_{2}^{\mathrm{bi}}}=0.05868$ and the corresponding minimal energy

$$
u_{\mathrm{bi}}^{\min }(B)=E_{0}^{\mathrm{bi}}-\frac{B^{2}}{4 E_{2}^{\mathrm{bi}}} .
$$




\section{Disaggregation onset}

The criterion for disaggregation onset in bilayers reads

$$
u_{\mathrm{bi}}^{\min }\left(B_{\mathrm{tr}}\right)=u_{\mathrm{gas}}=-B_{\mathrm{tr}} .
$$

By solving Eq. (B17), we find

$$
B_{\mathrm{tr}}=-u_{\mathrm{bi}}^{\min }\left(B_{\mathrm{tr}}\right)=3.206 \text {. }
$$

[1] K. De'Bell, A. B. MacIsaac, and J. P. Whitehead, Rev. Mod. Phys. 72, 225 (2000).

[2] J. J. Weis, J. Phys.: Condens. Matter 15, S1471 (2003).

[3] U. Gasser, J. Phys.: Condens. Matter 21, 203101 (2009).

[4] C.-Y. Chen and C.-S. Li, Phys. Fluids 22, 014105 (2010).

[5] J. Yan, S. C. Bae, and S. Granick, Adv. Mater. 27, 874 (2015).

[6] R. Seshadri and R. M. Westervelt, Phys. Rev. B 46, 5142 (1992).

[7] R. Seshadri and R. M. Westervelt, Phys. Rev. B 46, 5150 (1992).

[8] I. Musevic, Science 313, 954 (2006).

[9] W. Wen, F. Kun, K. F. Pal, D. W. Zheng, and K. N. Tu, Phys. Rev. E 59, R4758 (1999).

[10] J. Schockmel, E. Mersch, N. Vandewalle, and G. Lumay, Phys. Rev. E 87, 062201 (2013).

[11] R. Messina et al., Sci. Rep. 5, 10348 (2015).

[12] K. Zahn, R. Lenke, and G. Maret, Phys. Rev. Lett. 82, 2721 (1999).

[13] M. Golosovsky, Y. Saado, and D. Davidov, Appl. Phys. Lett. 75, 4168 (1999).

[14] W. Wen, L. Zhang, and P. Sheng, Phys. Rev. Lett. 85, 5464 (2000).

[15] G. Helgesen and A. T. Skjeltorp, J. Appl. Phys. 69, 8277 (1991).

[16] N. V. Dziomkina and G. J. Vancso, Soft Matter 1, 265 (2005).

[17] C. Ross, Annu. Rev. Mater. Res. 31, 203 (2001).

[18] Brown and J. A. Tuszynski, Ferroelectrics 220, 141 (1999).

[19] E. Rufeil-Fiori, N. Wilke, and A. J. Banchio, Soft Matter 12, 4769 (2016).

[20] J. J. Weis, Mol. Phys. 100, 579 (2002).

[21] J.-M. Caillol and J.-J. Weis, Mol. Phys. 113, 2487 (2015).

[22] H. Schmidle, C. K. Hall, O. D. Velev, and S. H. L. Klapp, Soft Matter 8, 1521 (2012).

[23] J. M. Tavares, J. J. Weis, and M. M. Telo da Gama, Phys. Rev. E 65, 061201 (2002)

[24] P. D. Duncan and P. J. Camp, J. Chem. Phys. 121, 11322 (2004).

[25] A. T. Skjeltorp, J. Appl. Phys. 57, 3285 (1985).

[26] K. Butter et al., Nat. Mater. 2, 88 (2003).

[27] A. Vorobiev, J. Major, H. Dosch, G. Gordeev, and D. Orlova, Phys. Rev. Lett. 93, 267203 (2004).

[28] J. Jordanovic and S. H. L. Klapp, Phys. Rev. Lett. 101, 038302 (2008).

[29] P. I. Belobrov, V. A. Voevodin, and V. A. Ignatchenko, Zh. Eksp. Teor. Fiz. 88, 889 (1985).

[30] J. Brankov and D. Danchev, Physica A 144, 128 (1987).

[31] S. Fazekas, J. Kertész, and D. E. Wolf, Phys. Rev. E 68, 041102 (2003).

[32] V. A. Froltsov, R. Blaak, C. N. Likos, and H. Löwen, Phys. Rev. E 68, 061406 (2003)

[33] We recall here that the dipole moments of an assembly of diploar particles on a planar surface are always in-plane in the ground-state. For a simple derivation, see R. Messina and I. Stanković, Europhys. Lett. 110, 46003 (2015).
[34] Bear in mind that in the absence of an applied magnetic field, the ground state of layered dipolar particles consists of stacked hexagonal lattices with (in-plane) ferromagnetic arrangement (i.e., all dipole moments point in the same coplanar direction $[43,44]$ ). Hence, a tangential (coplanar) magnetic field would only impose the chaining direction of the ground state without affecting the lattice symmetry.

[35] Monte Carlo simulations on dipolar bilayers, albeit without an external magnetic field and mainly focused on the liquid phase were reported in C. Alvarez, M. Mazars, and J.-J. Weis, Phys. Rev. E 77, 051501 (2008).

[36] S. W. de Leeuw, J. W. Perram, and E. R. Smith, Proc. R. Soc. A 373, 27 (1980).

[37] J.-J. Weis and D. Levesque, in Advanced Computer Simulation Approaches for Soft Matter Sciences II, edited by C. Holm and K. Kremer (Springer, Berlin, 2005), pp. 163-225.

[38] Steven G. Johnson, The NLOPT nonlinear-optimization package.

[39] R. Messina, L. A. Khalil, and I. Stankovic, Phys. Rev. E 89, 011202(R) (2014).

[40] G. Goldoni and F. M. Peeters, Phys. Rev. B 53, 4591 (1996).

[41] R. Messina and H. Löwen, Phys. Rev. Lett. 91, 146101 (2003).

[42] R. Tao and J. M. Sun, Phys. Rev. Lett. 67, 398 (1991).

[43] L. Spiteri and R. Messina, Phys. Rev. Lett. 119, 155501 (2017).

[44] L. Spiteri and R. Messina, Europhys. Lett. 120, 36001 (2017).

[45] It would be interesting to apply these ideas to monopolar bilayers and see what would be the effect of an imposed external electric field on the phase behavior of Wigner bilayers, see M. Antlanger, G. Kahl, M. Mazars, L. Samaj, and E. Trizac, Phys. Rev. Lett. 117, 118002 (2016).

[46] S. Sacanna, L. Rossi, and D. J. Pine, J. Am. Chem. Soc. 134, 6112 (2012).

[47] Y. Saado, M. Golosovsky, D. Davidov, and A. Frenkel, Phys. Rev. B 66, 195108 (2002).

[48] I. L. Lyubchanskii et al., J. Phys. D 36, R277 (2003).

[49] S. H. Im, Y. T. Lim, D. J. Suh, and O. O. Park, Adv. Mater. 14, 1367 (2002).

[50] M. E. Leunissen, H. R. Vutukuri, and A. van Blaaderen, Adv. Mater. 21, 3116 (2009).

[51] M. D. Cowley and R. E. Rosensweig, J. Fluid Mech. 30, 671 (1967).

[52] A. Gailīis, J. Fluid Mech. 82, 401 (1977).

[53] J. Lekner, Physica A 176, 485 (1991).

[54] A. Grzybowski and A. Brodka, Mol. Phys. 101, 1079 (2003).

[55] Note that this step necessitates either (i) a numerical resolution involving a fast and basic root finder algorithm or (ii) a Taylor expansion. In the latter case, the first terms for $\gamma(B)$ read $\gamma=\pi / 3+2.048 \times 10^{-3} B^{2}+7.394 \times$ $10^{-6} B^{4}+2.953 \times 10^{-8} B^{6}+O\left(B^{8}\right)$. 